\title{
Electric Field Effect on Excited State Binding Energy and Self-Polarization of a Hydrogenic Impurity in a Spherical Quantum Dot
}

\author{
Ali İhsan MESE ${ }^{1^{*}}$
}

\begin{abstract}
Effects of the electric field and impurity position on the 2p-excited state self-polarization and binding energy of the electron-impurity in a spherical quantum dot are investigated as a function of the dot radius using variational approach. According to results, turning point of $2 p$-excited state binding energy changes with the electric field strength and position of impurity in the spherical quantum dot. Self-polarization and binding energy between the 1s-ground state and 2p-excited state of a hydrogenic donor impurity in SQD are presented as a function of the dot radius for different electric field value and impurity positions. Obtained numerical results show a good agreement with the literature.
\end{abstract}

Keywords: Self-polarization, turning point, spherical dot, binding energy, electric field.

${ }^{1}$ Ali İhsan MESE (Orcid ID: 0000-0002-3901-590X), Trakya Üniversitesi, Fen Fakültesi, Fizik Bölümü, Edirne, Türkiye *Sorumlu Yazar/Corresponding Author: Ali İhsan MEŞE, e-mail: aliihsanmese@trakya.edu.tr 


\section{INTRODUCTION}

In the last decades, low dimensional structures (LDS) have attracted great interest because of their potential application to high performance (Bastard, 1981; Greene and Bajaj, 1985; Brown and Spector, 1986; Fraizzoli et al., 1990; Zhu et al., 1990; Montenegro and Merchancano, 1992; Branis et al., 1993; Johnson, 1995; Tsaousidou and Butcher, 1997; Brandi et al., 2002 Mese and Okan, 2004; Ulas et al., 2004; Esuanu et al., 2009; Baskoutas and Terzis, 2009). The main problems in LDS are conductivity of semiconductor and optical properties. The impurities play an important role in LDS because optical and electronic properties will be controlled. In some recent theoretical studies concern with different shape and different confinement potentials have shown that binding energy depends on the impurity positions (Chuu et al.,1992; Sucu et al., 2008; Sadeghi, 2009; Özmen, et al., 2009; Hassanabadi and Rajabi, 2009; Sadeghi and Rezaie, 2010; Rezaei et al., 2012; Sivakami and Gayathri, 2013; Kang et al., 2013; Wang et al., 2014). Spherical quantum dot (SQD) is very important because of its high symmetry which simplifies the theoretical calculations. It is noticed that recent theoretical studies have concentrated on the self-polarization and ground state binding energy. Self-polarization is defined as effect of confining potential on the donor impurity (Okan et al., 2004; Ulas et al., 2005; Erdogan et al., 2006; Erdogan et al., 2006; Akankan et al., 2006; Tangarife and Duque, 2011; Erdogan et al., 2013). However, there are no studies on the calculation of the $2 p$-state self-polarization depends on the different impurity positions in a GaAs/AlAs SQD under electric field.

In this work, self-polarization and binding energy between the 1s-ground state and $2 p$-excited state of a hydrogenic donor impurity in SQD are presented as a function of the dot radius for different electric field value and impurity positions using variational approach.

\section{MATERIALS AND METHODS}

Hamiltonian for an electron under the electric field effect is defined as (Erdogan et al., 2013)

$H=-\frac{\hbar^{2}}{2 \mathrm{~m}^{*}} \nabla^{2}+V(r)+\mathrm{eFr} \cos \theta$

$m^{*}$ is the electron effective mass and $\vec{F}$ is electric field and applied along the $\mathrm{z}$-axis. The confining potential is given by

$V(r)=\left\{\begin{array}{cc}0 & 0 \leq \mathrm{r} \leq \mathrm{R} \\ \infty & \mathrm{r}>\mathrm{R}\end{array}\right.$

The ground state energy and wave-function within the spherical dot $(n=1, l=0)$ is obtained by solution of the Schrodinger equation (Erdogan et al., 2013),

$\left[-\frac{\hbar^{2}}{2 \mathrm{~m}^{*}}\left(\frac{\mathrm{d}^{2}}{\mathrm{dr}^{2}}+\frac{2}{\mathrm{r}} \frac{\mathrm{d}}{\mathrm{dr}}+\frac{1}{\mathrm{r}^{2} \sin \theta} \frac{\partial}{\partial \theta} \sin \theta \frac{\partial}{\partial \theta}\right)+\mathrm{V}(\mathrm{r})+\mathrm{e} \operatorname{Fr} \cos \theta\right] \mathrm{R}_{10}^{0}(r, \theta)=E_{10}^{0} \mathrm{R}_{10}^{0}(r, \theta)$,

where upper subscript " 0 " refers to the subband. The eigenfunction, $\mathrm{R}_{10}^{0}(r, \theta)$, and corresponding energy eigenvalue is given by (Ghazi et al., 2013; Bulut et al., 2014; Mese et al., 2017),

$\mathrm{R}_{10}^{0}(r, \theta)=\left\{\begin{array}{lr}\mathrm{N}_{10}^{0} \frac{\sin (\alpha \mathrm{r})}{\mathrm{r}} \mathrm{e}^{-\beta \mathrm{rcos} \theta} & 0 \leq \mathrm{r} \leq \mathrm{R} \\ 0 & \mathrm{r}>\mathrm{R}\end{array}\right.$ 
$\alpha=\sqrt{2 m^{*} E_{10}^{0} / \hbar^{2}}$ and where ground state energy $E_{10}^{0}$ determined by minimization of the variational parameter $\beta$.

In the presence of impurity, the $2 p$-state energy of conduction subband of system is obtained by solving the Hamiltonian is given by,

$\left\{-\frac{\hbar^{2}}{2 \mathrm{~m}^{*}}\left[\frac{\partial^{2}}{\partial \mathrm{r}^{2}}+\frac{2}{\mathrm{r}} \frac{\partial}{\partial \mathrm{r}}+\frac{1}{\mathrm{r}^{2} \sin \theta} \frac{\partial}{\partial \theta} \sin \theta \frac{\partial}{\partial \theta}\right]+\mathrm{V}(\mathrm{r})+\mathrm{eFr} \cos \theta-\frac{\mathrm{e}^{2}}{4 \pi \varepsilon \varepsilon_{0}\left|\overrightarrow{\mathrm{r}}-\overrightarrow{\mathrm{r}}_{\mathrm{i}}\right|}\right\} \Psi_{21}(r, \theta)=E_{21}(r, \theta) \Psi_{21}(r, \theta)$

The 2 p-state impurity energy $E_{21}(r, \theta)$ is calculated by variational approach. The trial wave function can be defined as (Villamil and Montenegro, 1999; Villamil et al., 2005; Bulut et al., 2014; Mese et al., 2017),

$\Psi_{21}(r, \theta)=\left\{\begin{array}{ll}\mathrm{N}_{21} \frac{\sin (\alpha \mathrm{r})}{\mathrm{r}} \mathrm{r} \cos \theta \mathrm{e}^{-\beta \mathrm{rcos} \theta} \mathrm{e}^{-\lambda\left|\overrightarrow{\mathrm{r}}-\overrightarrow{\mathrm{r}}_{\mathrm{i}}\right|} & \mathrm{r}<\mathrm{R} \\ 0 & \mathrm{r} \geq \mathrm{R}\end{array}\right\}$

where, $r_{i}, N_{21}, \beta$ and $\lambda$ are impurity position which is along the $\mathrm{z}$ direction, normalization constant of $2 p$-excited state wave function and variational terms to include electric field and coulomb interaction, respectively.

The 2 p-state binding energy is given by with difference of 1 s ground state and $2 p$-excited state energy which defined as (Bulut et al., 2014),

$E_{b}(r, \theta)=E_{10}^{0}(r, \theta)_{\beta_{\text {min }}}-E_{21}(r, \theta)_{(\lambda, \beta)_{\min }}$

where, $E_{21}(r, \theta)_{(\lambda, \beta)_{\min }} 2$ p-state impurity energy determined by minimization of the variational parameters $\lambda, \beta$. (Sadeghi, 2009)

The self-polarization along the $\vec{r}_{i}$ direction for the 2 p-state can be defined as (Okan et al., 2004)

$\frac{S P}{e}=\frac{\left\langle\Psi_{21}(r, \theta)|| \overrightarrow{\mathrm{r}}-\overrightarrow{\mathrm{r}}_{\mathrm{i}}|| \Psi_{21}(r, \theta)\right\rangle}{\left\langle\Psi_{21}(r, \theta) \mid \Psi_{21}(r, \theta)\right\rangle}-\frac{\left\langle\Psi(r, \theta)|| \overrightarrow{\mathrm{r}}-\overrightarrow{\mathrm{r}}_{\mathrm{i}}|| \Psi(r, \theta)\right\rangle}{\langle\Psi(r, \theta) \mid \Psi(r, \theta)\rangle}$

$\Psi(r, \theta)$ indicates the wave function in the absence of SQD and defined as (Erdogan et al., 2013),

$\Psi(r, \theta)=\mathrm{Ne}^{-\left|\overrightarrow{\mathrm{r}}-\overrightarrow{\mathrm{r}}_{\mathrm{i}}\right| / 2 \mathrm{a}^{*}} \mathrm{r} \cos \theta$

\section{RESULTS AND DISCUSSION}

In this section, the $2 p$-state binding energy(EB), the $2 p$-state binging energy turning point (TP) and $2 p$-state self-polarization for various impurity position in the GaAs/AlAs SQD is calculated. The parameters used in this paper are; $\mathrm{m}^{*}=0.067 \mathrm{~m}_{0}$, effective Bohr radius $a^{*} \cong 100 A^{0}, \varepsilon=12.26$ and Rydgerg energy $R^{*}=5.83 \mathrm{meV}$. The results are presented in Fig.1-4.

In Fig. 1, the (BE) variation versus the dot radius for electric field strengths $F=(0,5$ and 10$) \mathrm{kV} / \mathrm{cm}$ in a SQD is presented. The results for the (TP) are in good agreement with $\mathrm{F}=0 \mathrm{kV} / \mathrm{cm}$ that reported 
(Bulut et al., 2014). As seen from the Fig.1 that the (BE) becomes negative at the different dot radius depends on electric filed which means that $2 p$-state unbounded. It means that, the $2 p$ impurity energy $\mathrm{E}_{21}$ is higher than the $1 \mathrm{~s}$ ground state energy $E_{10}^{0}$ with regard to our definition of binding energy. The (TP) is defined as the value of dot radius where the (EB) changes from negative to positive. It seen from the Fig. 1 that the (BE) becomes positive at radius values greater than $2.72 \mathrm{a}^{*}$ for $\mathrm{F}=0 \mathrm{kV} / \mathrm{cm}$ and $\mathrm{r}_{\mathrm{i}}=0$. Also, it can be seen that this result is in agreement with Refs. (Sadeghi and Rezaie, 2010). The small difference is due to the finite potential barrier. While the electric field strengths increase the (TP) shifts toward smaller dot radius. The turning point values for the electric field strengths $\mathrm{F}=(5$ and $\mathrm{F}=10) \mathrm{kV} / \mathrm{cm}$ have found as 2.29a* and 2.02a*, respectively. As seen Fig.1, Increasing the electric field strengths increases the positive (EB). It is quite the opposite according to the $1 \mathrm{~s}$ ground state.

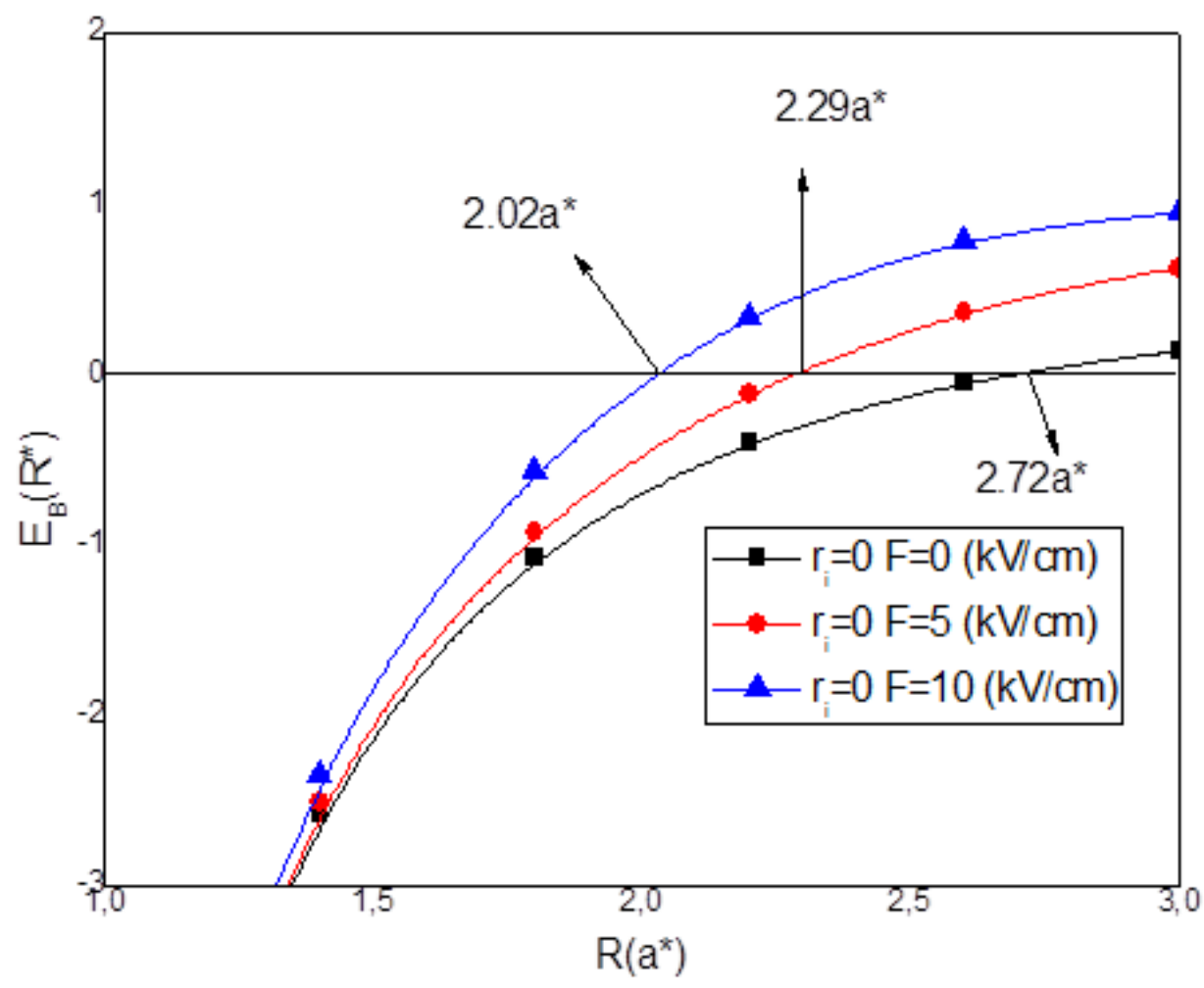

Figure 1.The $2 p$-state binding energy versus the dot radius for the electric field strengths $F=(0,5$ and 10$) \mathrm{kV} / \mathrm{cm}$ at impurity position $r_{i}=0$.

Fig. 2 depicts 2p-state self-polarization versus the dot radius for different electric field strengths. It is seen that the $2 p$-state self-polarization linearly increases with the electric field strengths. This is due to the fact that the growth of the electric field strengths reduces the potential of confinement and extends the areas of the wave function. As a result, self-polarization increases. This result is compatible with previous calculations (Erdogan et al., 2006).

The (TP) versus the dot radius for different impurity positions $\left(r_{i}=0, R / 2\right.$ and $R$ ) and electric field strengths $F=(0$ and 10$) \mathrm{kV} / \mathrm{cm}$ is presented in Fig.3. It is observed that (TP) decreases when the impurity shifts toward from centre to $\mathrm{r}_{\mathrm{i}}=\mathrm{R} / 2$ for $\mathrm{F}=0 \mathrm{kV} / \mathrm{cm}$. On the other hand, (TP) increases when the impurity shifts toward from $r_{i}=R / 2$ to edge of the quantum dot for electric field strength $F=0 \mathrm{kV} / \mathrm{cm}$. When the impurity shifts toward from centre to edge of the quantum dot for electric field strength $\mathrm{F}=10 \mathrm{kV} / \mathrm{cm}$ the (TP) increases. It is observed that electric field strength is effective on the (TP) value. This is due to the additional confinement cause by the electric field. 


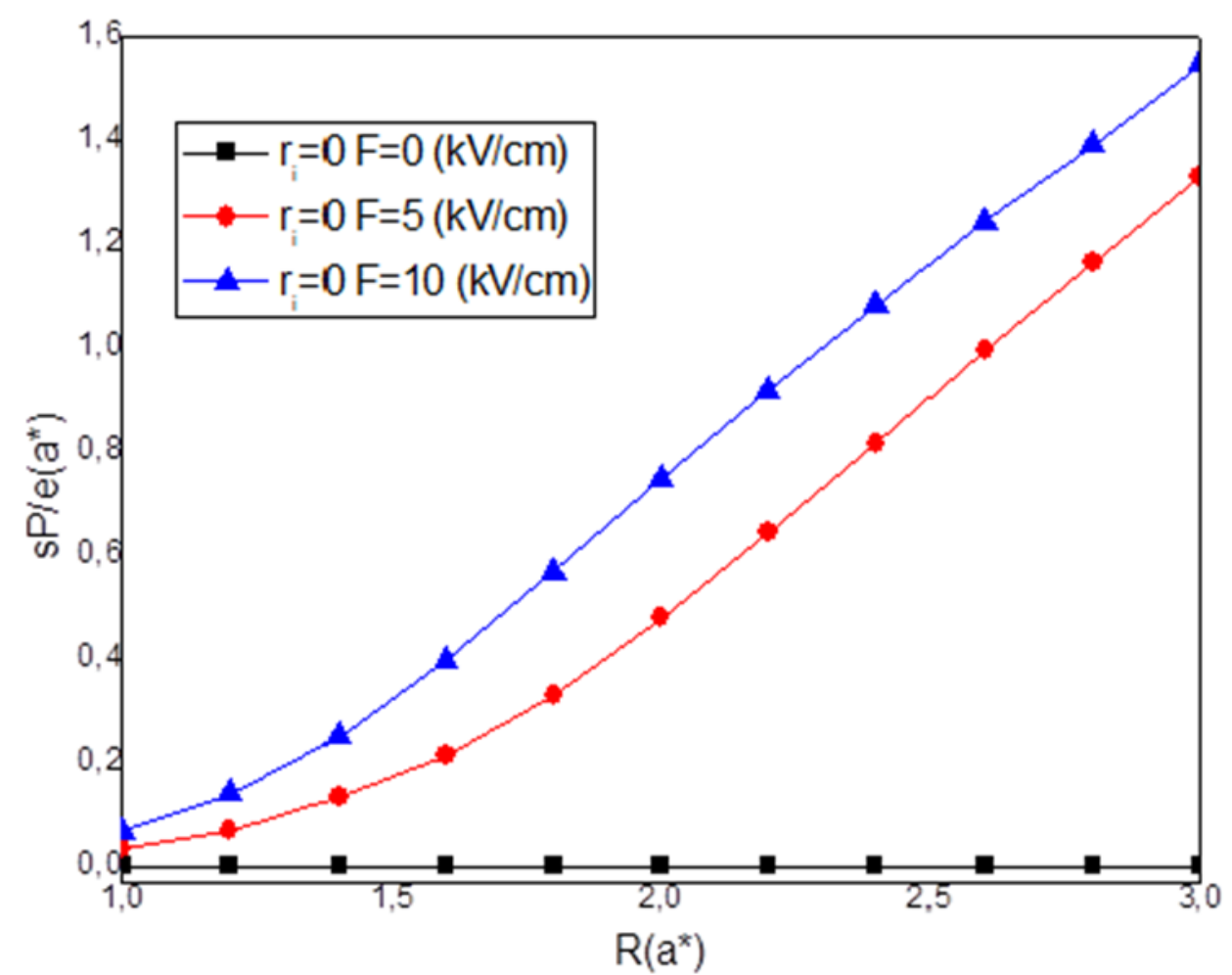

Figure 2. The $2 \mathrm{p}$-state self-polarization as a function of dot radius for same electric field strengths and impurity position with Figure 1.

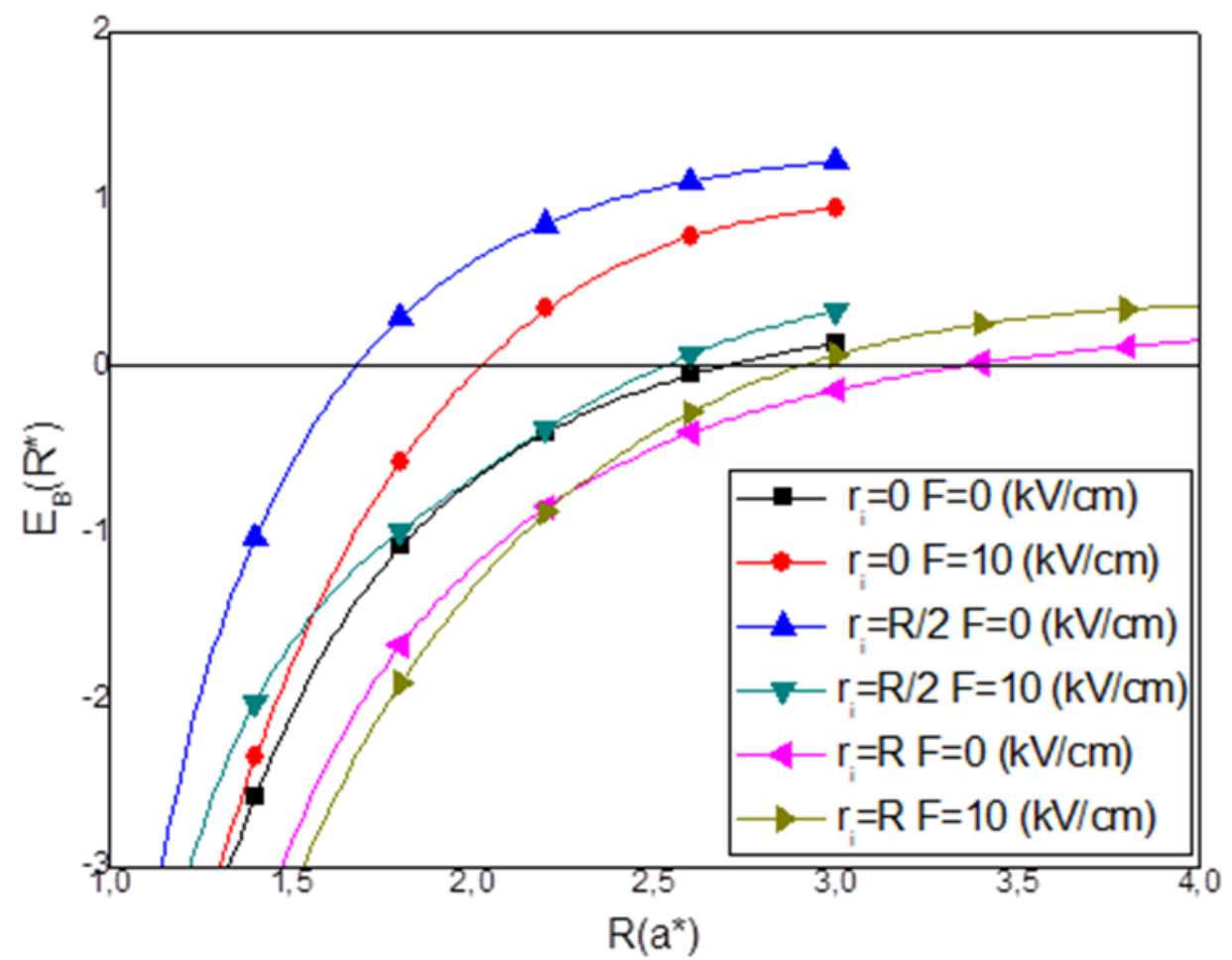

Figure 3. The $2 p$-state binding energy versus the dot radius for the electric field strengths $F=(0$ and 10$) \mathrm{kV} / \mathrm{cm}$ and impurity positions $r_{i}=0, r_{i}=R / 2$ and $r_{i}=R$. 


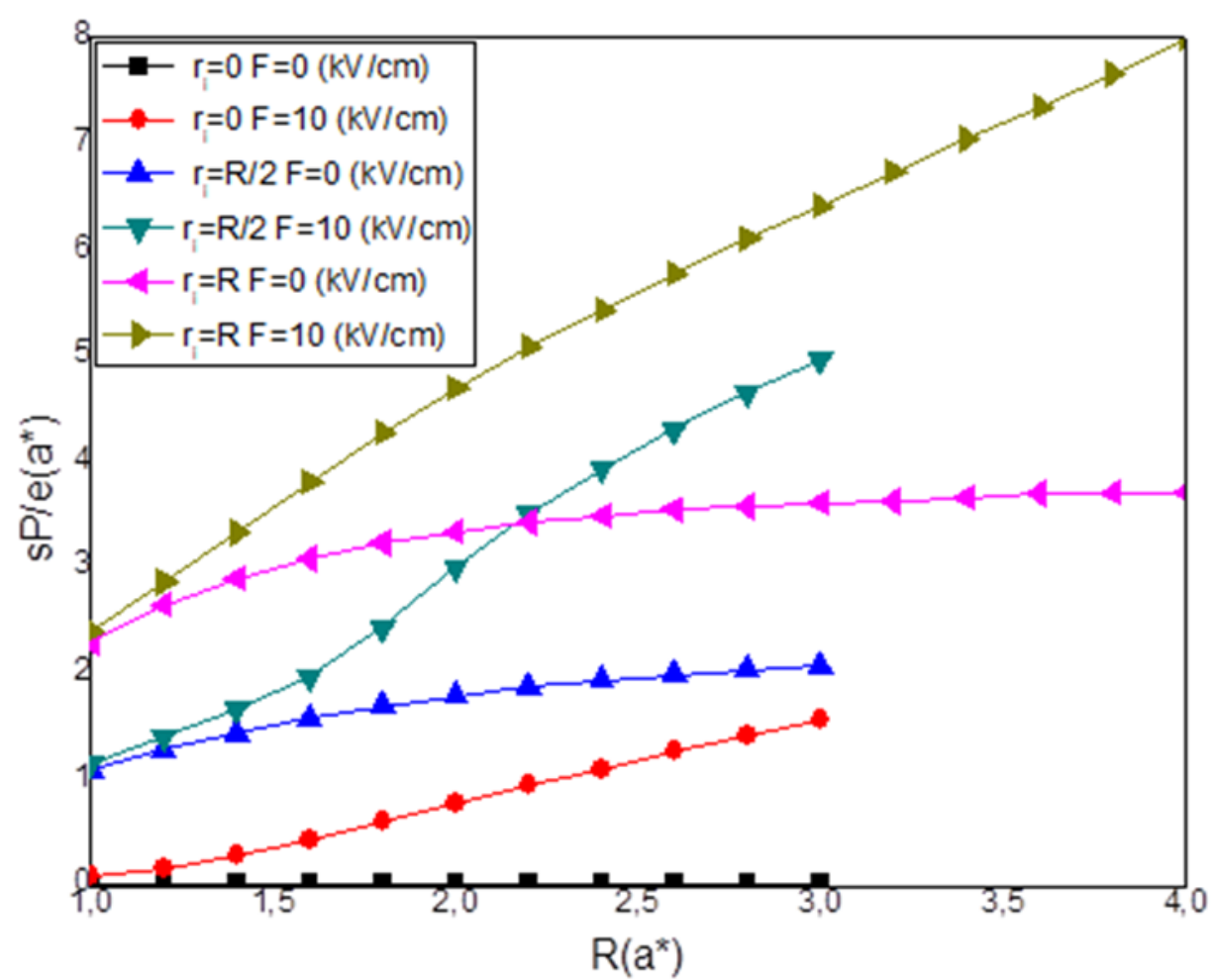

Figure 4. The $2 p$-state self-polarization versus dot radius for same electric field strengths and impurity positions with Figure 3.

To show the more effect of the impurity position on the $2 p$-state self-polarization, the $2 p$-state selfpolarization versus the dot radius for different impurity positions $\left(r_{i}=0, R / 2\right.$ and $\left.R\right)$ and electric field strengths $\mathrm{F}=(0$ and 10$) \mathrm{kV} / \mathrm{cm}$ has plotted in Fig. 4. As seen from the Fig.4, when the impurity shifts toward from centre to edge of the quantum dot for electric field strengths $\mathrm{F}=(0$ and 10$) \mathrm{kV} / \mathrm{cm}$, the $2 \mathrm{p}-$ state self-polarization approximately linearly increases. For very large quantum dots, the results tend towards to three-dimensional bulk case (Bulut et al., 2014; Mese et al., 2017).

\section{CONCLUSION}

The effects of impurity position and electric field strength on the (TP) versus dot radius are calculated. The results show that the effects of electric field and impurity position are very important on (TP). The first time $2 \mathrm{p}$-state self-polarization in spherical quantum dot has been calculated.

\section{REFERENCES}

Akankan O, Erdogan I, Akbas H, 2006. Spatial electric field effect on the self-polarization in GaAs/AlAs square quantum-well wires. Physica E, 35 (1): 217-221.

Baskoutas S, Terzis AF, 2009. Binding energy of hydrogenic impurity states in an inverse parabolic quantum well under static external fields. The European Physical Journal B, 69: 237-244.

Bastard G, 1981. Hydrogenic impurity states in a quantum well: A simple model. Physical Review B, 24(8): 4714-4722.

Bella RSD, Navaneethakrishnan K, 2004. Donor binding energies and spin-orbit coupling in a spherical quantum dot. Solid State Communications, 130 (11): 773-776.

Brandi HS, Latge A, Oliveira LE, 2002. Magnetic-field and laser effects on the electronic and donor states in semiconducting quantum dots. Journal of Applied Physics, 92 (8): 4209-4213.

Branis SV, Li G, Bajaj KK, 1993. Hydrogenic impurities in quantum wires in the presence of a magnetic field. Physical Review B, 47 (3): 1316-1323. 
Brown JW, Spector HN, 1986. Hydrogen impurities in quantum well wires. Journal of Applied Physics, 59 (4): 1179-1186.

Bulut P, Erdogan I, Akbas H, 2014. Binding energy of 2p-bound state of a hydrogenic donor impurity in a GaAs/Ga1_xAlxAs spherical quantum dot under hydrostatic pressure. Physica E, 63: 299303.

Chuu DS, Hsiao CM, Mei WN, 1992. Hydrogenic impurity states in quantum dots and quantum wires. Physical Review B, 46 (7): 3898-3905.

Erdogan I, Akankan O, Akbas H, 2006. Electric and magnetic field effects on the self-polarization in GaAs/AlAs cylindrical quantum well-wires. Physica E, 33 (1): 83-87.

Erdogan I, Akankan O, Akbas H, 2006. Binding energy and self-polarization as function of energy density in GaAs/AlAs quantum well wires. Physica E, 35 (1): 27-32.

Erdogan I, Akankan O, Akbas H, 2013. Simultaneous effects of temperature, hydrostatic pressure and electric field on the self-polarization and electric field polarization in a $\mathrm{GaAs} / \mathrm{Ga}_{0.7} \mathrm{Al}_{0.3} \mathrm{As}$ spherical quantum dot with a donor impurity. Superlattices and Microstructures, 59: 13-20.

Esuanu N, Niculescu EC, Bureleanu LM, 2009. Simultaneous effects of pressure and laser field on donors in GaAs/Ga1-x $\mathrm{Al}_{\mathrm{x}} \mathrm{As}$ quantum wells. Physica E, 41 (8): 1386-1392.

Fraizzoli S, Bassani F, Buczko R, 1990. Shallow donor impurities in GaAs-Ga1-x $\mathrm{Al}_{\mathrm{x}}$ As quantum-well structures: Role of the dielectric-constant mismatch. Physical Review B, 41 (8): 5096-5103.

Ghazi HE, Jorio A, Zorkani I, 2013. Impurity binding energy of lowest-excited state in (In,Ga)N-GaN spherical QD under electric field effect. Physica B, 426: 155-157.

Greene RL, Bajaj KK, 1985. Binding energy of the $2 \mathrm{p}_{0}$-like level of a hydrogenic donor in GaAs-Ga $\mathrm{Ga}_{1-\mathrm{x}}$ $\mathrm{Al}_{\mathrm{x}}$ As quantum-well structures. Physical Review B, 31 (6): 4006-4008.

Hassanabadi H, Rajabi AA, 2009. Energy levels of a spherical quantum dot in a confining potential. Physics Letters A, 373 (6): 679-681.

Johnson NF, 1995. Quantum dots: few-body, low-dimensional systems. Journal of Physics: Condensed Matter, 7 (1): 965-989.

Kang S, Yang YC, He J, Xiong FQ, Xu N, 2013. The hydrogen atom confined in both Debye screening potential and impenetrable spherical box. Central European Journal of Physics, 11 (5): 584-593.

Mese AI, Okan SE, 2004. Binding energy of relativistic hydrogenic impurities in cylindrical quantum well wires under an applied electric field. Physica Status Solidi B, 241 (15): 3525-3531.

Mese AI, Cicek E, Erdogan E, Akankan O, Akbas H, 2017. The effect of dielectric constant on binding energy and impurity self-polarization in a GaAs-Ga1-xAlxAs spherical quantum dot. Indian Journal of Physics, 91(3): 263-268.

Montenegro NP, Merchancano STP, 1992. Hydrogenic impurities in GaAs-(Ga,Al)As quantum dots. Physical Review B, 46(15): 9780-9783.

Okan SE, Erdogan I, Akbas H, 2004. Anomalous polarization in an electric field and self-polarization in GaAs/AlAs quantum wells and quantum well wires. Physica E, 21 (1): 91-95.

Özmen A, Yakar Y, Çakır B, Atav Ü, 2009. Computation of the oscillator strength and absorption coefficients for the intersubband transitions of the spherical quantum dot. Optics Communications, 282 (19): 3999-4004.

Rezaei G, Mousavi S, Sadeghi E, 2012. External electric field and hydrostatic pressure effects on the binding energy and self-polarization of an off-center hydrogenic impurity confined in a GaAs/AlGaAs square quantum well wire. Physica B, 407 (13): 2637-2641. 
Sivakami A, Gayathri V, 2013. Hydrostatic pressure and temperature dependence of dielectric mismatch effecton the impurity binding energy in a spherical quantum dot. Superlattices and Microstructures, 58: 218-227.

Sadeghi E, 2009. Impurity binding energy of excited states in spherical quantum dot. Physica E, 41 (7): 1319-.1322

Sadeghi E, Rezaie GH, 2010. Effect of magnetic field on the impurity binding energy of the excited states in spherical quantum dot. Pramana-Journal of Physics, 75: 749-755.

Sucu S, Mese AI, Okan SE, 2008. The role of confinement and shape on the binding energy of an electron in a quantum dot. Physica E, 40 (8): 2698-2702.

Tangarife E, Duque CA, 2011. Simultaneous effects of hydrostatic pressure and electric field on impurity binding energy and polarizability in coupled InAs/GaAs quantum wires. Physica B, 406 (4): 952956.

Tsaousidou M, Butcher PN, 1997. Phonon-drag thermopower of a ballistic quantum wire Physical Review B, 56 (16): R10044-R10047.

Ulas M, Cicek E, Dalgic SS, 2004. Electric field effect on the binding energy of a non-hydrogenic donor impurity in a cylindrical cross-sectional quantum well wire. Physica Status Solidi B, 241 (13): 2968-2974.

Ulas M, Erdogan I, Cicek E, Senturk Dalgic S, 2005. Self-polarization in GaAs-(Ga, Al)As quantum well wires: electric field and geometrical effects. Physica E, 25 (4): 515-520.

Villamil PV, Montenegro NP, 1999. Excited-States and Infrared Transition Energies of a Donor Impurity in a Disc-Shaped GaAs Quantum Dot under the Action of an Applied Magnetic Field. Journal of Physics: Condensed Matter, 11: 9723-9730.

Villamil PV, Cabra C, Montenegro NP, 2005. Shallow Inpurity and Transition Energies in Cylindirical GaAs-Ga0.6A10.4As Quantum Well Wires under Applied Magnetic Field. . Journal of Physics: Condensed Matter, 17 (): 5049-5058.

Wang S, Kang Y, LiLi X, 2014. Binding energy of the ground and first fewexcited states of hydrogenic donor impurityin a rectangular GaAs quantum dot in the presenceof electric field. Superlattices and Microstructures, 76: 221-233.

Zhu JL, Xiong JJ, Gu BL, 1990. Confined electron and hydrogenic donor states in a spherical quantum dot of GaAs-Ga1-xAlxAs. Physical Review B, 41 (9): 6001-6007. 